\title{
Capsule Commentary on Carey et al., Is Non-steroidal Anti-inflammatory Therapy Non-inferior to Antibiotic Therapy in Uncomplicated Urinary Tract Infections: a Systematic Review
}

\author{
Sol Aldrete, MD \\ Medical College of Wisconsin, Wauwatosa, WI, USA. \\ J Gen Intern Med 35(6): 1936 \\ DOI: $10.1007 / \mathrm{s} 11606-020-05758-6$ \\ (c) Society of General Internal Medicine 2020
}

$\mathrm{U}$ rinary tract infections (UTIs) are among the most common bacterial infection in the community and account for more than 8 million office visits annually, including emergency department and urgent care visits. ${ }^{1}$ As antibiotic resistance continues to emerge worldwide, identifying antibiotic sparing agents becomes more relevant every day.

Carey et al. ${ }^{2}$ performed a systematic review of the literature with the goal to answer the question of whether non-steroidal anti-inflammatory therapy (NSAIDs) can be used in the management of uncomplicated UTIs instead of antibiotic therapy. They included five randomized clinical trials with 1309 women and looked at symptoms resolution as their primary outcome and at antibiotic prescription and rate of pyelonephritis as secondary outcomes. Their findings support the use of antibiotics as first-line treatment for uncomplicated UTI for both symptom resolution and prevention of pyelonephritis.

Diagnosis of a UTI is usually based on systemic or localized symptoms in conjunction with a positive urine culture; however, the inclusion criteria for all five randomized trials were based only on symptoms typical for UTI without requiring a positive urine culture. This raises the concern that we might be overcalling the diagnosis and missing other potential etiologies, as this study by Shapiro et al. ${ }^{3}$ found were out of 92 women enrolled in a prospective cohort study presenting to an urban emergency department with urinary complaints, a total of $57.3 \%$ had positive urine cultures and $17.3 \%$ also had a sexually transmitted infection. Additionally, almost half of the participants (39-58\%) in the NSAIDs group achieved resolution of their symptoms by day 3 or 4 which raises the question if these were patients that were symptomatic for another reason or had asymptomatic bacteriuria and did not require antibiotic therapy at all.

For clinicians, this study confirms the importance of antibiotics in the management of uncomplicated UTIs but also highlights the importance of obtaining a detailed history, urinalysis, urine culture, and pelvic examination when indicated to arrive to the appropriate diagnosis and target antimicrobial therapy better. Future studies should continue to evaluate antibiotic sparing agents (e.g., vitamin C, probiotics) as we continue to encounter increasing antimicrobial resistance.

Corresponding Author: Sol Aldrete, MD; Medical College of Wisconsin, Wauwatosa, WI, USA (e-mail: saldrete@mcw.edu).

Compliance with Ethical Standards:

Conflict of Interest: No conflict of interest.

\section{REFERENCES}

1. Foxman B. The epidemiology of urinary tract infection. Nat Rev Urol 2010;7(12):653-60

2. Carey MR, Vaughn VM, Mann J, Townsend W, Chopra V, Patel PK. Is Non-Steroidal Anti-Inflammatory Therapy Non-Inferior to Antibiotic Therapy in Uncomplicated Urinary Tract Infections: A Systematic Review. J Gen Intern Med. https://doi.org/10.1007/s11606-020-05745-x.

3. Shapiro T, Dalton M, Hammock J, Lavery R, Matjucha J, Salo DF. The prevalence of urinary tract infections and sexually transmitted disease in women with symptoms of a simple urinary tract infection stratified by low colony count criteria. Acad Emerg Med 2005;12(1):38-44.

Publisher's Note Springer Nature remains neutral with regard to jurisdictional claims in published maps and institutional affiliations.

Published online March 3, 2020 